\title{
Domain Structures Across the Martensitic Transformation
}

\section{in $\mathrm{Ni}_{2+\mathrm{x}} \mathrm{Mn}_{1-\mathrm{x}} \mathrm{Ga}$}

\section{Deepti Jain ${ }^{a}$, Soma Banik ${ }^{b}$, L.S.Sharath Chandra ${ }^{c}$, S.R.Barman ${ }^{d}$, R.Nath $^{\mathrm{e}}$ and V.Ganesan ${ }^{f}$}

UGC-DAE consortium for Scientific Research, University campus, Khandwa Road, Indore (MP) 452001, India

adeeptijain25@gmail.com, bsomabanik@csr.ernet.in, csharath_ls@csr.ernet.in, dbarman@csr.ernet.in, ${ }^{\mathrm{e}}$ rnath@csr.ernet.in, ${ }^{\mathrm{f}} \mathrm{vg}$ anesan@csr.ernet.in

\section{Keywords: $\mathrm{Ni}_{2} \mathrm{MnGa}$, Optical Microscopy, MFM, Ferromagnetic Shape memory alloys}

\begin{abstract}
Evolution of domain structures across the martensitic transition $\left(\mathrm{T}_{\mathrm{m}}\right)$ in the ferromagnetic shape memory alloy system Ni-Mn-Ga is studied using an optical microscope with a temperature variation. Compositions chosen have $\mathrm{T}_{\mathrm{m}}<\mathrm{T}_{\mathrm{c}}, \mathrm{T}_{\mathrm{m}}=\mathrm{T}_{\mathrm{c}}$ and $\mathrm{T}_{\mathrm{m}}>\mathrm{T}_{\mathrm{c}},\left(\mathrm{T}_{\mathrm{c}}=\right.$ Curie temperature) so that one can compare the nature of martensitic domains. There are no appreciable domain structures when $T_{m}<T_{c}$ as compared to the one with $T_{m}>T_{c}$. However, giant morphological changes in the form of appearance of well-developed domains that are propagating with different directions are seen for the composition in which $\mathrm{T}_{\mathrm{m}}=\mathrm{T}_{\mathrm{c}}$. The results are discussed in the light of Magnetic Force Microscopy observations as well as giant entropy changes known to occur on samples with cooccurrence of $\mathrm{T}_{\mathrm{m}}$ and $\mathrm{T}_{\mathrm{c}}$.
\end{abstract}

\section{Introduction}

Ferromagnetic shape memory alloys are technologically important materials. They are also important in understanding variety of phenomena in basic physics. These alloys show two transitions, martensitic and Curie in nature. $\mathrm{Ni}_{2} \mathrm{MnGa}$ is an interesting ferromagnetic shape memory alloy that has attracted enormous attention in recent times due its unique magneto-elastic properties and the large magnetic field induced strain, which attracts the technological motive [1-4]. Detailed investigations on the crystal structure of $\mathrm{Ni}_{2+x} \mathrm{Mn}_{1-\mathrm{x}} \mathrm{Ga}$ have been studied as a function of composition and a phase diagram has been established $[5,6]$. The technical aspects of the alloys are understood in terms of domains that are formed as a consequence of free energy minimization and their dynamics in terms of thermodynamic variable like temperature and magnetic field. Ferroelastic and ferromagnetic domain structures are quite familiar in these materials and their knowledge is an important component to further these materials to technological applications. Field and temperature dependant evolution of micromagnetic structures have been studied in detail by many authors with diverse techniques. Bitter decoration with a DIC technique was employed to unravel this evolution of magnetic and twin domains [7, 8] and a visual evidence for its magneto-elastic coupling [9]. Lorentz microscopy has revealed a clear herring bone martensitic domain structures and stripe domains that are attributed to the orientation of the easy axis of the magnetization with respect to the sample surface [10]. Magnetic Force Microscopy (MFM) has been extensively used to investigate the domains in $\mathrm{Ni}_{2} \mathrm{MnGa}$. Surface relief seen at zero field, wiping out of such relief at moderate fields of $\sim 2 \mathrm{kOe}$, Fir tree patterns, fir patterns localized at twin boundaries, single domains and a de-twined interior on higher fields etc are few important observations [11-14]. In a recent investigation we have reported the MFM studies on samples of this family where in the evolution of twin and Weiss type domains are reported across the Curie transition [15]. Optical microscopy along with MFM mapping on coupled influence of martensitic and ferromagnetic transition on the magnetic domain structures in $\mathrm{Ni}_{2} \mathrm{MnGa}$ is reported here. The samples studied are from $\mathrm{Ni}_{2+\mathrm{x}} \mathrm{Mn}_{1-\mathrm{x}} \mathrm{Ga}$ family with $\mathrm{x}=0.13,0.22$ and 0.35 such that $\mathrm{T}_{\mathrm{m}}<\mathrm{T}_{\mathrm{c}}, \mathrm{T}_{\mathrm{m}}=\mathrm{T}_{\mathrm{c}}$ and $\mathrm{T}_{\mathrm{m}}>\mathrm{T}_{\mathrm{c}}$. 


\section{Experimental}

Polycrystalline samples of $\mathrm{Ni}_{2+\mathrm{x}} \mathrm{Mn}_{1-\mathrm{x}} \mathrm{Ga}(\mathrm{x}=0.13,0.22$ and 0.35$)$ were prepared using argon arc furnace melting. Samples are then annealed at $1100 \mathrm{~K}$ for 9days and followed by quenching in ice water. XRD, EDAX, DSC and magnetic susceptibility have been used to characterize the samples $[5,6]$. Final composition is determined by EDAX and transition temperature obtained by DSC and magnetic susceptibility is as follows: $\mathrm{Ni}_{2.13} \mathrm{Mn}_{0.87} \mathrm{Ga}_{1.02}\left(\mathrm{~T}_{\mathrm{m}} \sim 291 \mathrm{~K}, \mathrm{~T}_{\mathrm{c}} \sim 336 \mathrm{~K}\right), \mathrm{Ni}_{2.22} \mathrm{Mn}_{0.78} \mathrm{Ga}$ $\left(\mathrm{T}_{\mathrm{m}}=\mathrm{T}_{\mathrm{c}} \sim 334 \mathrm{~K}\right)$ and $\mathrm{Ni}_{2.35} \mathrm{Mn}_{0.65} \mathrm{Ga}$. $\left(\mathrm{T}_{\mathrm{m}} \sim 536 \mathrm{~K}, \mathrm{~T}_{\mathrm{c}} \sim 320 \mathrm{~K}\right)$. Room temperature $\mathrm{XRD}$ at various compositions of $\mathrm{Ni}_{2+x} \mathrm{Mn}_{1-\mathrm{X}} \mathrm{Ga}$ describes the austenite cubic phase with space group $\mathrm{Fm} 3 \mathrm{~m}$ and unmodulated martensitic tetragonal phase with space group I4/mmm [15]. Optical micrograph is obtained using Carl Zeiss LSM 510 Meta optical microscope with 20x objective. Linkam cooling/heating stage is attached to the microscope to study the temperature induced phase transition. Magnetic Force Microscopy (MFM) imaging was carried out at different temperatures using multimode Nanoscope-IVa from Veeco-Digital Instruments USA with a high temperature attachment option.

\section{Results and Discussion}

We have studied the evolution of martensitic domains across the martensitic transition using optical microscopy while the magnetic Weiss domains across the Curie transition was studied using MFM. We have studied the magnetic and martensitic domains structure in various phases like ferromagnetic martensitic and paramagnetic martensite etc. Figure 1 shows the evolution of microstructure at various temperatures observed by optical microscopy and magnetic force microscopy for the sample $\mathrm{Ni}_{2.22} \mathrm{Mn}_{0.78} \mathrm{Ga}$, in which martensitic and curie transition temperature coincide. Even though the data is taken at various intervals of temperature, we limit here our discussion with only few of them. Sample is polished at room temperature, which means that polishing is done at ferromagnetic martensitic phase. Due to this it is expected that the morphology is featureless as in Figure $1 \mathrm{a}$ and when heated across the $\mathrm{Tm}$ it will develop features that corresponds to the surface relief due to martensite- austenite transition as in Figure $1 \mathrm{~b}$. Figure $1 \mathrm{~b}$ is quite aesthetic in the sense that it reveals different variant configurations separated by habit planes. The white protrusions are sample irregularities, which were used as markers. In single crystalline samples these habit planes used to run over several hundreds of microns and one could see a clear single austenite - martensite phase boundary running through the sample and can be modeled clearly as a habit plane [7]. Due to the polycrystalline nature of the sample, the habit planes are restricted to smaller length $(\sim 100-200 \mu \mathrm{m})$. There is a reason to justify this from figure $1 \mathrm{c}$ where in a closer view of one set of twins that splits in to branches before crossing the region of narrower twin bands is shown. A regular rectangular twin bands of width $\sim 25-40 \mu \mathrm{m}$ and length $\sim 200 \mu \mathrm{m}$ is shown in figure $1 \mathrm{~d}$ along with its section in figure $1 \mathrm{e}$.

Figure $1 \mathrm{f}$ shows the topography of the MFM measurements, where in we again see a featureless image at room temperature due to the polishing effects. However, the corresponding MFM image (figurelf-right image) shows clear and contrasting magnetic domains structures. The larger and rectangular domains seen can be classified as martensitic twins, which have a periodicity of 12-15 $\mu \mathrm{m}$ and are $>100 \mu \mathrm{m}$ long. They are seen in MFM because they are also ferromagnetic in nature. Inside these martensitic twins, clear sub domain structures are seen which are attributed to Weiss type domains. These fine structures are quite clear and are zig-zag in nature and are similar to the observations reported earlier [7]. The almost uniformly spaced magnetic Weiss domains terminate at the interface of the martensitic domains. This is in line with the optical and MFM observation of micro magnetic structure in $\mathrm{Ni}_{2} \mathrm{MnGa}[5,9,11]$. Appearance of surface relief of twins type domains is observed in this sample at temperature $T>T_{c}, T_{m}(334 K)$ i.e at paramagnetic austenite phase and are shown in figures $1 \mathrm{~b}, 1 \mathrm{c}, 1 \mathrm{~d}$. The periodicity of this relief is varies $10 \mathrm{um}$ to $25 \mathrm{um}$. This relief is also appearing at the place of martensitic twins as clear from the left side topography of the MFM image in figure1g. Its right frame, MFM, is featureless because one has entered inside the 
paramagnetic state and hence the magnetic signal is vanished. If one does a Fourier transform of the MFM image of Figure 1f and AFM image of Figure 1g, their periods are same. The effects are pronounced only on this sample where $T_{m} \sim T_{c}$. Giant entropy change at the co-occurrence of structural and magnetic transition in $\mathrm{Ni}_{2.19} \mathrm{Mn}_{0.81} \mathrm{Ga}$ alloy has been reported by Pareti et al [17]. They could observe a magnetic entropy change of $20 \mathrm{~J} / \mathrm{kgK}$ when transition are co-occurring while it is only $5 \mathrm{~J} / \mathrm{kgK}$ when only structural transition occur, for a field span of 0 to $1.6 \mathrm{~T}$. A magnetically driven reverse transformation temperature $\left(140^{\circ} \mathrm{C} / \mathrm{T}\right)$ has been observed in $\mathrm{Ni}_{53} \mathrm{Mn}_{25} \mathrm{Ga}_{22}$ by Jiang et al [16] at a low field of $50 \mathrm{mT}$ which is about 2 orders of magnitude grater then the value of $1^{\circ} \mathrm{C} / \mathrm{T}$ observed for $\mathrm{Ni}_{2} \mathrm{MnGa}$. They have attributed this giant change to the co occurrence of magnetic and structural transition in the former. Comparing the above said giant changes, it is also believed that giant morphological features seen in our specific sample where in the $T_{m}$ and $T_{c}$ coincides may be correlated to the effect of co-occurrence.

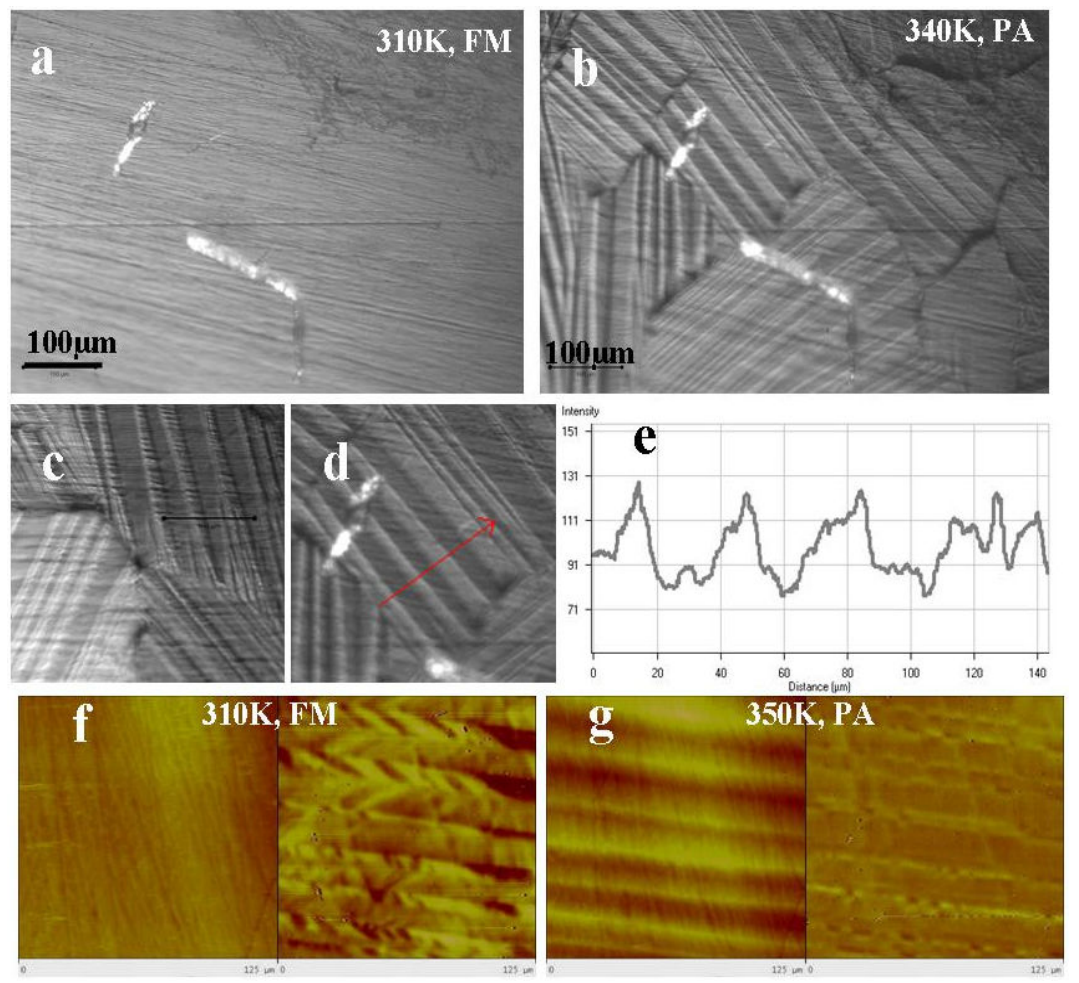

Fig.1: Microstructures of $\mathrm{Ni}_{2.22} \mathrm{Mn}_{0.78} \mathrm{Ga}\left(\mathrm{T}_{\mathrm{m}}=\mathrm{T}_{\mathrm{c}} \sim 334 \mathrm{~K}\right.$ ) by optical microscope (a-e) and MFM (f$\mathrm{g})$. Images (a) and left frame of (f) are at ferromagnetic martensitic phase and are featureless due to polishing effects. Surface relief of twins domains structure at $\mathrm{T}=340 \mathrm{~K}$ (b)-(d), Intensity profile of arrow indicated in image (d) is shown in (e). AFM (left) and MFM(right), 125 $\mu$ images of sample at ferromagnetic martensitic (FM) (f) and paramagnetic austenite (PA)phase (g).MFM has contrasting features like twin and weiss domains that disappear after Tc. AFM which is featureless shows strong surface reliefs while crossing $\mathrm{Tm}$ which also coincides with $\mathrm{T}_{\mathrm{c}}$ in this sample.

Figure 2 shows optical and MFM microscopy results on sample $\mathrm{Ni}_{2.35} \mathrm{Mn}_{0.65} \mathrm{Ga}\left(\mathrm{T}_{\mathrm{m}} \sim 536 \mathrm{~K}\right.$, $\left.\mathrm{T}_{\mathrm{c}} \sim 320 \mathrm{~K}\right)$ with room temperature ferromagnetic martensitic phase. As usual the room temperature images (figure $2 \mathrm{a}$ and left of $2 \mathrm{e}$ ) are featureless except scratches due to polishing. The corresponding MFM image (right of 2e) shows the twin type magnetic domain structure with periodicity 18-20um, which is expected and in line with the literature [11]. Upon crossing Tm, imaged at $550 \mathrm{~K}$, one could see a clear evolution of Martensitic bands in optical images (2b). Sample is in paramagnetic austenite phase when heated above 536K. Surface reliefs due to selfaccommodating twin variants are clearly seen in figure $2 \mathrm{~b}$. The sizes of the twin domains vary from region to region with dagger shaped structures. Figure $2 c$ shows a magnified portion of $2 b$, wherein 
the domains widths are larger. A careful observation also reveals transverse sub domains or internal twins running at $90^{\circ}$ to the main bands and may be attributed to the magnetic structures. The observed are in line with the literature [18]. These morphological features are less intense as compare to first sample because it evolves only by structural transition. MFM image above 330K shows the paramagnetic nature of the sample.
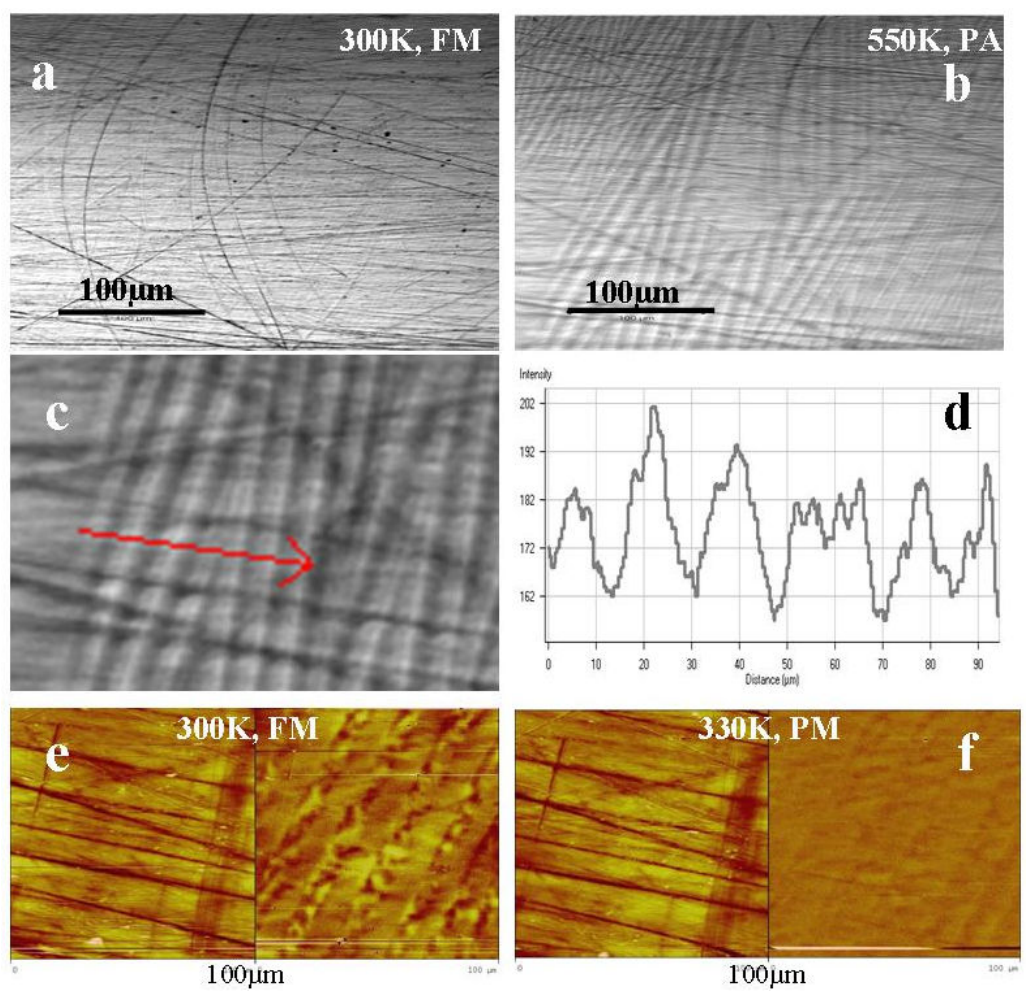

Fig. 2: Optical and MFM images of $\mathrm{Ni}_{2.35} \mathrm{Mn}_{0.65} \mathrm{Ga}\left(\mathrm{T}_{\mathrm{m}} \sim 536 \mathrm{~K}, \mathrm{~T}_{\mathrm{c}} \sim 320 \mathrm{~K}\right)$. a) Optical image at Martensitic and b) Austenite phase where in clear evolution of twin structures in the form of surface relief is seen, c) A closer view of bottom left portion of b), d) its line profile. e) and f) are the MFM images below and above $\mathrm{T}_{\mathrm{c}}$. FM means Ferromagnetic Martensitic, PA means Paramagnetic Austenite, PM=Paramagnetic Martensitic.
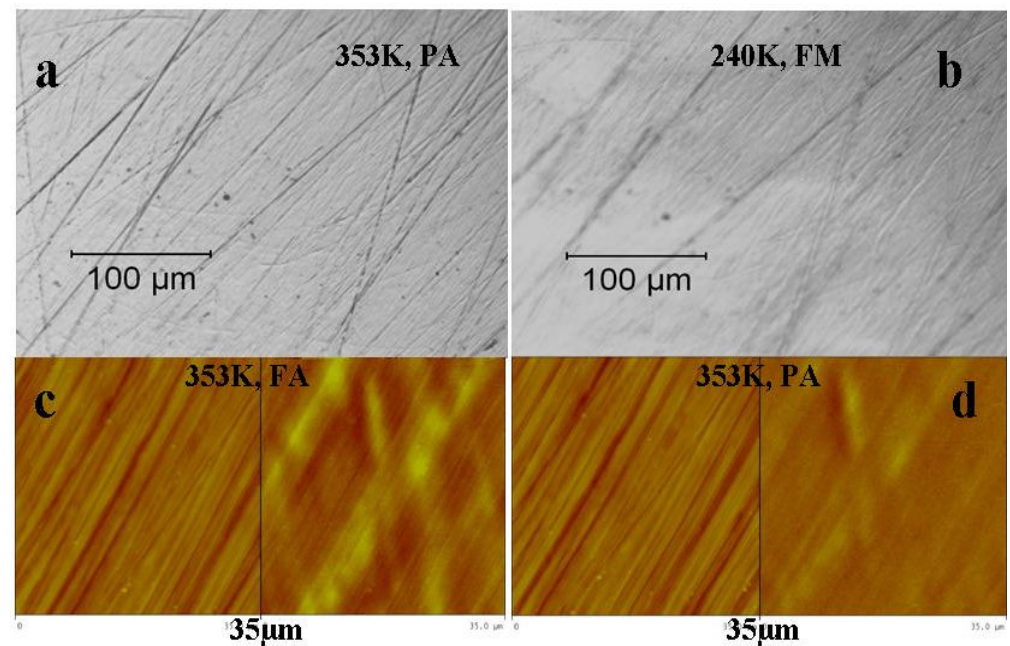

Fig. 3: Optical and MFM micrographs of $\mathrm{Ni}_{2.13} \mathrm{Mn}_{0.87} \mathrm{Ga}_{1.02}\left(\mathrm{~T}_{\mathrm{m}} \sim 291 \mathrm{~K}, \mathrm{~T}_{\mathrm{c}} \sim 336 \mathrm{~K}\right)$. a) and b) are optical images at Austenite and Martensitic phase. c) and d) are MFM images below and above $\mathrm{T}_{\mathrm{c}}$. FM=Ferromagnetic Martensitic, PA=Paramagnetic Austenite, FA= Ferromagnetic Austenite. 
The next sample we have chosen for study is $\mathrm{Ni}_{2.13} \mathrm{Mn}_{0.87} \mathrm{Ga}_{1.02}$ which has transition temperatures $\mathrm{T}_{\mathrm{m}} \sim 291 \mathrm{~K}, \mathrm{~T}_{\mathrm{c}} \sim 336 \mathrm{~K}$ and ferromagnetic austenite phase at room temperature. Figure 3 shows the evolution of microstructure at various temperatures observed by optical microscope and MFM for the sample $\mathrm{Ni}_{2.13} \mathrm{Mn}_{0.87} \mathrm{Ga}_{1.02}$. As usual the sample was polished at room temperature and hence the surface is featureless at room temperature in both AFM and optical images ( $3 \mathrm{a} \& 3 \mathrm{c}$ ). MFM image at room temperature shows random structures of magnetic domains as shown in figure $3 \mathrm{c}$ as expected in ferromagnetic austenite phase. Magnetic domains disappeared on heating in its paramagnetic phase (figure 3d). One can expect the appearance of martensitic twins while cooling of this sample, because in general the fcc-fct transformation results in complex martensitic structure caused by the simultaneous nucleation and growth of a martensitic band across the length of the sample and their subsequent intersection with each other as reported in literature [7]. Surprisingly we are unable to observe any formation of twin type structures at the sample surface as shown in figure $3 \mathrm{~b}$. This may be attributed to the mismatch between the grain orientations of our sample to that of crystallographic variant such that we are unable to pick up the domains.

\section{Conclusion:}

We have studied the evolution of domain structures in samples of $\mathrm{Ni}_{2} \mathrm{MnGa}$ where in $\mathrm{T}_{\mathrm{c}}=\mathrm{T}_{\mathrm{m}}, \mathrm{T}_{\mathrm{c}}<\mathrm{T}_{\mathrm{m}}$ and $T_{c}>T_{m}$. Clear and giant morphological features changes is observed in optical as well as in MFM for samples where $\mathrm{Tm} \sim \mathrm{Tc}$ as compare to $\mathrm{Tm}>\mathrm{Tc}$ and is attributed to the giant entropy changes due to co-occurrence effects. Clear changes associated with martensitic transition are seen when the sample is in the paramagnetic state $\left(\mathrm{T}_{\mathrm{c}}<\mathrm{T}_{\mathrm{m}}\right)$. No observable twin structures are seen when the Tm lies in the ferromagnetic state $T_{m}<T_{c}$, and attributed to the mismatch between the grain orientations that of crystallographic variant.

\section{Acknowledgements:}

Authors would like to thank Dr. P. Chaddah and Prof. Ajay Gupta for their encouragement and the staff of the low temperature laboratory especially Mr. Mohan Gangrade for their interest. DJ \& LSSC would like to thank CSIR; India for their SRF and DJ would like to thank DST India for a financial grant for attending this ICFSMA09 conference as well as the conference organisers for their hospitality.

\section{References:}

[1] S. J. Murray, M. Marioni, S. M. Allen, R. C. O’handley, and T. A. Lograsso: Appl. Phys. Lett. Vol. 77 (2000), p. 886

[2] A. Sozinov, A. A. Likhachev, N. Lanska, and K. Ullakko: Appl. Phys. Lett. Vol. 80 (2002), p. 1746

[3] J. Marcos, L. Mañosa, A. Planes, F. Casanova, X. Batlle, and A. Labarta: Phys. Rev. B Vol. 68 (2003), p. 094401

[4] C. M. Craciunescu, and M. Wuttig: J. Optoelectron. Adv. Mat. Vol. 5 (2003), p. 139

[5] S. Banik, R. Ranjan, A. Chakrabarti, S. Bhardwaj, N. P. Lalla, A. M. Awasthi, V. Sathe, D. M. Phase, P. K. Mukhopadhyay, D. Pandey, and S. R. Barman: Phys. Rev. B Vol. 75 (2007), p. 104107

[6] S. Banik, A. Chakrabarti, U. Kumar, P. K. Mukhopadhyay, A. M. Awasthi, R. Ranjan, J. Schneider, B. L. Ahuja and S. R. Barman: Phys. Rev. B Vol. 74 (2006), p. 085110

[7] M R. Sullivan and H. D. Chopra: Phys. Rev. B Vol. 70 (2004), p. 094427

[8] M R. Sullivan A. A. Shah, and H. D. Chopra: Phys. Rev. B Vol. 70 (2004), p. 094428

[9] H. D. Chopra, Chunhai ji, and V. V. Kokorin: Phys. Rev. B Vol. 61 (2000), p. R14913

[10] S.P.Venkateswaran, N.T. Nuhfer, and M.de Graef: Acta Materialia, Vol. 55, (2007), p. 5419

[11] Qi Pan, and R. D. James: J. Appl. Phys. Vol. 87 (2000), p. 4702 
[12] Qi Pan, J. W. Dong, C. J. Palmstrom, J. Cui and R. D. James: J. Appl. Phys. Vol. 91 (2002), p. 7812

[13] V. A. Chernenko, R L. Anton, M. Kohl, M. Ohtsuka, I Orue, and J. M. Barandiaran: J. Phys.: Condens. Matter, Vol. 17 (2005), p. 5215

[14] C. Biswas, S. Banik, A. K. Shukla, R. S. Dhaka,V. Ganesan, and S. R. Barman: Surf. Sci. Vol. 600 (2006), p. 3749

[15] Deepti Jain, Soma Banik, L.S.Sharath Chandra, S.R.Barman, R.Nath and V. Ganesan: Adv. Mate. Res. Vol. 52 (2008), p. 115

[16] C. Jiang, G. Feng, and H. Xu: Appl. Phys. Lett. Vol. 80 (2002), p. 1619

[17] L. Pareti, M. Solzi, F. Albertini, and A. Paoluzi: Eur. Phys. J. B Vol. 32 (2003), p. 303

[18] Y.Ge, O.Heczko, O.Soderberg and V.K.Lindroos: J. Appl. Phys. Vol. 96 (2004), p. 2159 